\title{
Genetic and pathologic characteristics of gastrointestinal stromal tumors in extragastric lesions
}

\author{
SONGDE CHO $^{1}$, YASUHIKO KITADAI ${ }^{1}$, SHIGETO YOSHIDA ${ }^{1}$, SHINJI TANAKA ${ }^{2}$, \\ MASAHARU YOSHIHARA $^{3}$, KAZUHIRO YOSHIDA $^{4}$ and KAZUAKI CHAYAMA ${ }^{1}$ \\ ${ }^{1}$ Department of Medicine and Molecular Science, Hiroshima University Graduate School of Biomedical Sciences, \\ 1-2-3 Kasumi, Minami-ku; ${ }^{2}$ Department of Endoscopy, Hiroshima University Hospital, Hiroshima; \\ ${ }^{3}$ Health Service Center, Hiroshima University, Higashi-Hiroshima; ${ }^{4}$ Department of Oncology, \\ Research Institute for Radiation Biology and Medicine, Hiroshima University, Hiroshima, Japan
}

Received June 2, 2006; Accepted August 18, 2006

\begin{abstract}
The goal of this study was to investigate differences in the clinicopathologic and genetic characteristics of gastric and extragastric gastrointestinal stromal tumors (GISTs). We evaluated 13 extragastric GISTs and compared them with 56 gastric GISTs, which were described previously. DNA was extracted from paraffin-embedded tumor specimens, and exons $9,11,13$, and 17 of the KIT gene and exons 12 and 18 of the platelet-derived growth factor receptor $\alpha$ (PDGFRA) gene were amplified by polymerase chain reaction and sequenced. Immunohistochemistry was performed for KIT, CD34, Ki-67 (as a marker of cell proliferation), and CD31 (as a marker of microvessel density), and apoptosis was assessed by in situ DNA nick end-labeling. Of the 13 extragastric GISTs $7(54 \%)$ had a mutation in exon 11 of KIT, and $2(15 \%)$ had a mutation in exon 13 of KIT. Deletions in exon 11 of KIT were the most common mutation encountered in the extragastric GISTs. The extragastric GISTs, especially small intestinal GISTs, showed larger deletions, leading to deletions of amino acid residues in the KIT protein, and higher vascularity than did the gastric GISTs. These data suggest that extragastric GISTs differ from gastric GISTs with respect to associated mutations and angiogenic activity.
\end{abstract}

\section{Introduction}

Gastrointestinal stromal tumor (GIST) is the most common mesenchymal tumor of the human gastrointestinal (GI) tract.

Correspondence to: Dr Yasuhiko Kitadai, Department of Medicine and Molecular Science, Hiroshima University Graduate School of Biomedical Sciences, 1-2-3 Kasumi, Minami-ku, Hiroshima 734-8551, Japan

E-mail: kitadai@hiroshima-u.ac.jp

Key words: gastrointestinal stromal tumor, KIT, platelet-derived growth factor receptor $\alpha$, mutation, deletion, extragastric, small intestine
Although stromal tumors can occur throughout the GI tract from the esophagus to the rectum, they occur most frequently in the stomach (1), less frequently in the small intestine, and least often at other sites. GISTs differ from other mesenchymal tumors histologically, immunohistochemically, and genetically (2). Only GISTs express KIT, a proto-oncogene protein, and expression of KIT has been reported in $89-100 \%$ of GISTs $(3,4)$. Sequencing of the KIT gene has revealed the presence of activating mutations in many GISTs (5). The KIT gene encodes a receptor-type tyrosine kinase that is composed of an intracellular tyrosine kinase, a juxtamembrane (JM) region, and an extracellular domain with a ligand-binding site. The KIT ligand, stem cell factor (SCF), binds to KIT and causes receptor dimerization, activation of kinase activity, and autophosphorylation (6). KIT mutants that undergo autophosphorylation in the absence of SCF can contribute to the growth of GISTs (5). Sommer et al (7) showed that a knock-in $K I T$-activating mutation was sufficient to induce development of GISTs in mice. It has been reported that a subset of GISTs that lacked KIT mutations had activating mutations in a related receptor tyrosine kinase, platelet-derived growth factor receptor $\alpha$ (PDGFRA) (8). Immunostaining of PDGFRA has proved to be a helpful marker for discriminating $K I T$ negative GISTs from other gastrointestinal mesenchymal lesions (9).

The KIT and PDGFRA genes belong to the family of class III receptor protein tyrosine kinases (RTKs), which also includes the colony-stimulating factor I receptor, PDGFRß, and FMS-related tyrosine kinase 3 (10). KIT and PDGFRA mutations have been found only in GISTs; cancers of other histologic types lacked such mutations (11).

We previously reported correlations of mutations of the KIT and PDGFRA genes in gastric GISTs with cell proliferation, angiogenesis, and apoptosis (12). In the present study, we screened 13 extragastric GISTs for mutations in exons 9, 11, 13, and 17 of KIT and in exons 12 and 18 of PDGFRA and also examined cell proliferation, angiogenesis, and apoptosis in extragastric GISTs. We compared the clinicopathologic and genetic characteristics of gastric GISTs with those of extragastric GISTs. 
Table I. Clinicopathologic and genetic data of GISTs in the extragastric GISTs.

\begin{tabular}{|c|c|c|c|c|c|c|c|c|}
\hline No. & Age & Sex & Location & $\begin{array}{l}\text { Tumor size } \\
(\mathrm{cm})\end{array}$ & Mutation & Ki-67 LI & MVD & $\begin{array}{l}\text { Apoptotic } \\
\text { index }\end{array}$ \\
\hline 1 & 70 & M & Small bowel & 11.0 & KIT exon 13 & 2.5 & 15 & 0.6 \\
\hline 2 & 25 & M & Small bowel & 9.0 & KIT exon 13 & 1.5 & 18 & 0.2 \\
\hline 3 & 79 & M & Small bowel & 2.0 & Wild-type & 0.6 & 27 & 0.2 \\
\hline 4 & 63 & M & Small bowel & 9.0 & KIT exon 11 & 7.8 & 23 & 1.4 \\
\hline 5 & 62 & $\mathrm{~F}$ & Small bowel & 3.5 & Wild-type & 0.6 & 28 & 0.2 \\
\hline 6 & 45 & $\mathrm{~F}$ & Small bowel & 8.5 & KIT exon 11 & 5.0 & 20 & 0.6 \\
\hline 7 & 64 & M & Small bowel & 1.5 & Wild-type & 2.5 & 18 & 1.0 \\
\hline 8 & 37 & $\mathrm{~F}$ & Small bowel & 7.0 & KIT exon 11 & 3.5 & 25 & 0.5 \\
\hline 9 & 64 & M & Small bowel & 4.0 & KIT exon 11 & 3.0 & 20 & 0.2 \\
\hline 10 & 76 & M & Small bowel & 5.5 & KIT exon 11 & 3.0 & 24 & 0.6 \\
\hline 11 & 63 & $\mathrm{~F}$ & Rectum & 5.0 & KIT exon 11 & 0.3 & 18 & 0.4 \\
\hline 12 & 55 & $\mathrm{~F}$ & Rectum & 10.0 & KIT exon 11 & 0.5 & 18 & 0.6 \\
\hline 13 & 71 & M & Rectum & 6.0 & Wild-type & 0.2 & 6 & 0.7 \\
\hline
\end{tabular}

Ki-67 LI, Ki-67 labeling index; MVD, microvessel density.

\section{Materials and methods}

Patients and tumor specimens. We retrospectively analyzed the clinical records of 16 patients who underwent surgical resection for mesenchymal tumor of the GI tract other than the stomach at Hiroshima University Hospital during the period 1981-2004. All patients presented with a solitary tumor. Mesenchymal tumors were identified as GISTs on the basis of positive immunohistochemical staining for KIT and/or CD34. Of the 16 tumors (81\%) 13 were identified as GISTs. None of the 13 patients was treated with STI571 before surgery. For strict privacy protection, identifying information for all samples was removed prior to analysis; the procedure was in accordance with the Japanese Government's Ethics Guidelines for Human Genome/Gene Research.

Immunohistochemistry. Expression of KIT, CD34, Ki-67, and CD31 was analyzed by immunohistochemistry. A representative tissue block was collected for each case, and immunohistochemistry was performed on $4 \mu \mathrm{m}$-thick sections of formalin-fixed, paraffin-embedded tissues cut from these blocks. KIT and CD34 were stained with the EnVision System (Dako Cytomation, Carpinteria, CA), and Ki-67 and CD31 were examined with the LSAB2 kit (Dako Cytomation) as described previously (12). The primary antibodies were antiKIT (diluted 1:50; Dako Cytomation), anti-CD34 (Histofine, Tokyo, Japan), anti-Ki-67 (MIB-1, diluted 1:50; Dako Cytomation) and anti-CD31 (Dako Cytomation).

The Ki-67-labeling index (LI) was determined by light microscopy of the area with the greatest number of Ki-67positive cells. These areas were identified by scanning tumor sections at low power (x40 and x100). The Ki-67 LI was calculated as the percentage of Ki-67-positive cells in 1,000 tumor cells examined.

Microvessel density (MVD) was determined by light microscopy examination of CD31 staining of the area with the
Table II. Correlation between clinicopathologic/genetic features and locations of GISTs.

\begin{tabular}{llll}
\hline Location & Stomach $^{\mathrm{a}}$ & $\begin{array}{c}\text { Small bowel/ } \\
\text { rectum }\end{array}$ & p-value \\
\hline Number of cases & 56 & 13 & \\
KIT expression (\%) & $51(91)$ & $12(92)$ & 0.887 \\
CD34 expression (\%) & $44(79)$ & $10(77)$ & 0.897 \\
Sex (male/female) & $35 / 21$ & $8 / 5$ & 0.949 \\
Age, years (range) & $60(27-86)$ & $60(25-79)$ & 0.849 \\
Mutation positive $(\%)$ & $43(76)$ & $9(69)$ & 0.569 \\
Tumor size (cm) & 4.5 & 6.3 & 0.086 \\
Ki-67 LI (\%) & 2.2 & 2.4 & 0.761 \\
MVD & 14.9 & 20.0 & 0.049 \\
Apoptotic index $(\%)$ & 0.59 & 0.55 & 0.783 \\
\hline
\end{tabular}

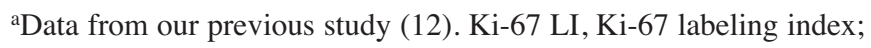
MVD, microvessel density.

greatest number of capillaries and small venules. Areas of high vascularity were identified by scanning of tumor sections at low power (x40 and $\mathrm{x} 100)$. Vessels were counted in the three areas of a x400 field (x40 objective and x10 ocular; $0.189 \mathrm{~mm}^{2}$ per field) with the greatest neovascularization, and the average count was noted.

In situ DNA nick end-labeling. DNA strand breaks due to apoptosis were detected in situ by terminal deoxynucleotidyl transferase-mediated dUTP-biotin nick end-labeling (TUNEL) with a commercial kit (ApopTag, Chemicon, Temecula, CA). This is based on the specific binding of terminal deoxynucleotidyl transferase to 3'-OH ends of DNA. We used this 


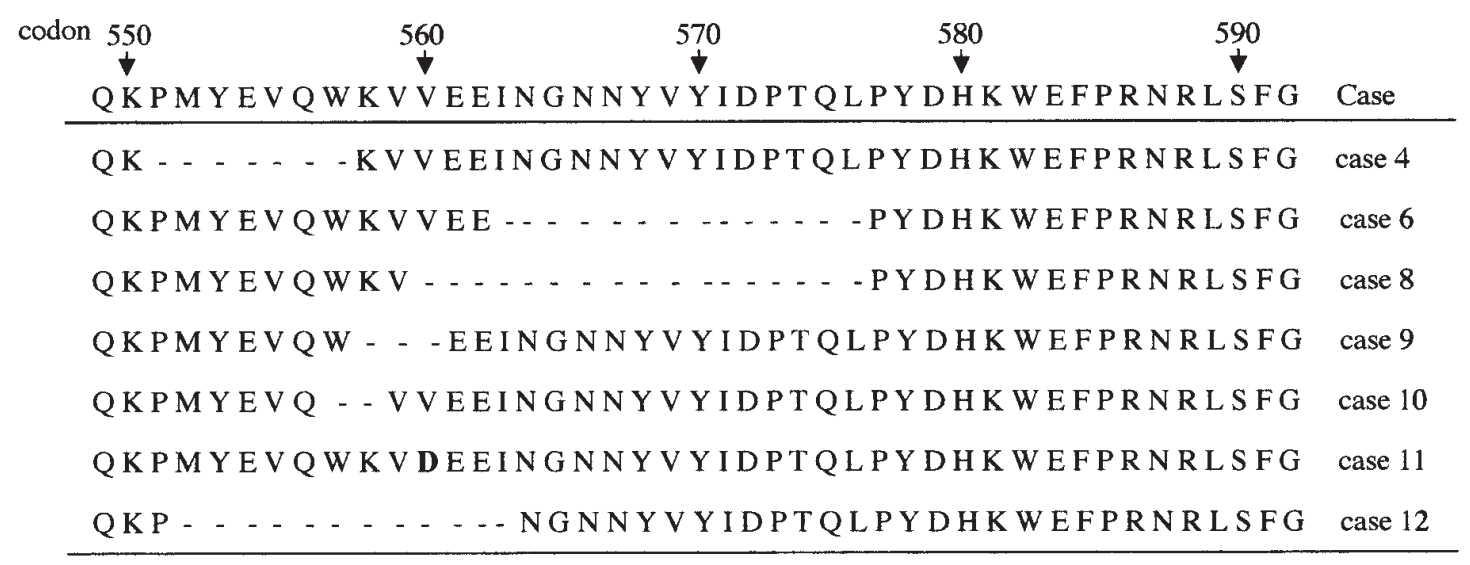

Figure 1. Predicted amino acid sequences of KIT with exon 11 mutations. The sequence starts at codon 549 and ends at codon 592 . The wild-type sequence is shown on top. Blanks (---) correspond to deletions, and amino acid substitutions are indicated in bold type.

method to detect apoptotic cells in $4 \mu$ m-thick sections of formalin-fixed, paraffin-embedded GISTs as described previously (12). The percentage of TUNEL-positive apoptotic cells in approximately 1,000 tumor cells was calculated.

Molecular analysis. Genomic DNA was extracted from formalin-fixed, paraffin-embedded GIST tissues. In all cases, exons $9,11,13$, and 17 of the KIT gene and exons 12 and 18 of the PDGFRA gene were evaluated for mutations by polymerase chain reaction (PCR) amplification and direct sequencing of the amplification products as described previously (12). To verify sequences, we performed direct sequencing of amplicons from two independent PCRs of the same template DNA. Sequencing was performed with an ABI PRISM 310 DNA sequencer (Applied Biosystems, Foster City, CA).

Statistical methods. To evaluate the relations between locations of GISTs and clinicopathologic/genetic features, data were analyzed by $\chi^{2}$ or Fisher's exact test in cross tables and by Student's t-test. The significance level was set at $\mathrm{p}<0.05$ for each analysis.

\section{Results}

Clinicopathologic features. We examined 13 extragastric GISTs comprising 10 small intestinal and 3 rectal tumors. No GISTs were derived from esophagus. The clinicopathologic features of the extragastric GISTs examined in the present study are shown in Table I. Comparisons of clinicopathologic data of extragastric GISTs with those of gastric GISTs, which were previously published (12), are shown in Table II. Of the 13 extragastric GISTs, 10 (77\%) expressed both KIT and CD34, 2 (15\%) expressed only KIT, and $1(8 \%)$ expressed only CD34. The patients from whom these GISTs were obtained comprised of 8 men and 5 women, and the mean age of the patients at the time of surgical resection was 60 years (range, 25-79 years). The mean tumor size of extragastric GISTs was $6.3 \mathrm{~cm}$ (range, $1.5-11.0 \mathrm{~cm}$ ), which was larger than that of gastric GISTs, although the difference was not statistically significant.
Evaluation of mutations. Mutations were detected in 9 (69\%) of 13 extragastric GISTs (Table I). Seven (54\%) of these mutations were in exon 11 and two (15\%) were in exon 13 of $K I T$. We did not detect any mutations in exons 9 and 17 of the KIT gene or exons 12 and 18 of the PDGFRA gene. None of the analyzed tumors contained more than one of these mutations.

Of 7 GISTs that had mutations in exon 11 of the KIT gene, 6 contained simple deletions, and 1 carried a simple substitution (Fig. 1). Deletions contained 6-51 bps, which led to deletions of 2-17 amino acid residues from the KIT protein. None of these mutations disrupted the downstream open reading frame of the gene. In other words, all of exon 11 deletions in the present study were in-frame deletions. In all six cases, the deletions contained sequences between codons 551 and 576. The only substitution was V560D, which occurred in a mutational 'hot-spot' (2). Internal tandem duplications at the 3 ' end of exon 11 , which has also been reported as a mutational 'hot spot' in GISTs, especially those in the stomach (13), were not found in the extragastric GISTs examined in the present study.

Two mutations, K642E and M651V, were detected in exon 13 of the KIT gene. Mutations detected in exon 13 of the KIT gene are shown in Fig. 2. Mutations in exon 17 of the KIT gene are quite rare in GISTs $(14,15)$. In the present study, no patient carried a mutation in exon 17. In all 9 extragastric GISTs with mutations, we detected wild-type $K I T$, indicating that only 1 of the 2 KIT alleles was mutated in these GIST samples.

Correlation between clinicopathologic/genetic features and locations of GISTs. The relations between clinicopathologic features and locations of GISTs are shown in Table II. KIT was expressed in $91 \%$ of gastric GISTs and in $92 \%$ of extragastric GISTs. CD34 was expressed in 79\% of gastric GISTs and 77\% of extragastric GISTs. There were no differences between gastric GISTs and extragastric GISTs with respect to sex ratio or patient age. We found that $76 \%$ of gastric GISTs and $69 \%$ of extragastric GISTs carried mutations. Extragastric GISTs tended to be larger in size than gastric GISTs, although the difference was not statically significant. A significant 


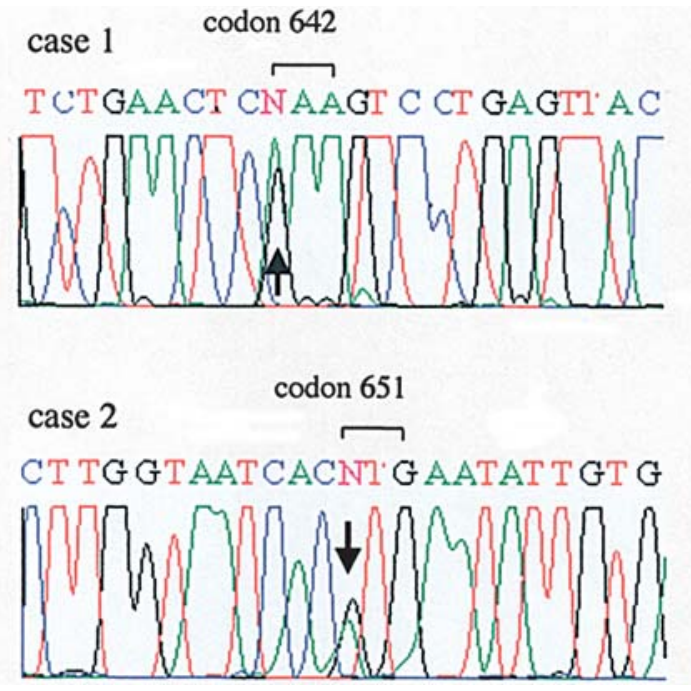

Figure 2. Direct sequencing of mutant KIT exon 13 from GISTs. Case 1, arrow indicates heterozygous point mutation $(A \rightarrow G)$ at the first nucleotide position of codon 642, resulting in the K642E substitution. Case 2, arrow indicates a heterozygous point mutation $(A \rightarrow G)$ at the first nucleotide position of codon 651 , resulting in the M651V substitution.

difference was detected in MVD as assessed with CD31 (Fig. 3). Microvessels were more abundant in extragastric GISTs than in gastric GISTs ( $\mathrm{p}=0.049)$. When we compared the 9 small intestinal (extragastric) GISTs with the 56 gastric GISTs, the significance of the difference increased $(\mathrm{p}=0.017)$. Ki-67 LI and apoptotic index showed no correlation with GIST location.

\section{Discussion}

The frequency of GISTs with mutations varied widely in previous studies $(14,16-18)$. Most mutations have been found in exon 11 of the KIT gene, which contains a mutational 'hotspot' (2). In the present study, we detected mutations in exon 11 of the KIT gene in 54\% (7/13) of extragastric GISTs. Of the 7 GISTs with exon 11 mutations, 6 contained deletions, and only 1 mutation was a substitution. KIT exon 11 mutations were detected in 5 of 10 small intestinal GISTs, and all 5 mutations were deletions. We previously reported that 34 of 56 gastric GISTs (61\%) harbored mutations in exon 11 of the KIT gene; 20 cases contained deletions, and 14 cases had only substitutions (12). Therefore, deletions in exon 11 of the KIT gene appear to be more common than simple substitutions in extragastric GISTs. In our previous study, deletions in gastric GISTs comprised of 1-6 amino acid residues (12). In the present study, the deletions detected in the 6 extragastric GISTs comprised of 2-17 amino acid residues, and the average number of deleted amino acids was 9.2. The mean number of amino acid residues deleted from KIT was larger in extragastric GISTs than in gastric GISTs ( $\mathrm{p}=0.0001$ ), and the mean number of those was larger in small intestinal GISTs than in gastric GISTs ( $p=0.0007$, Student's t-test).

The frequency of mutations in exon 9 of the KIT gene has been reported to be much lower than that of mutations in exon 11. As reported previously, mutations in exon 9 consist of identical tandem repeats of sequences encoding Ala-Tyr, which corresponds to codons 502 and $503(16,19)$. GISTs with

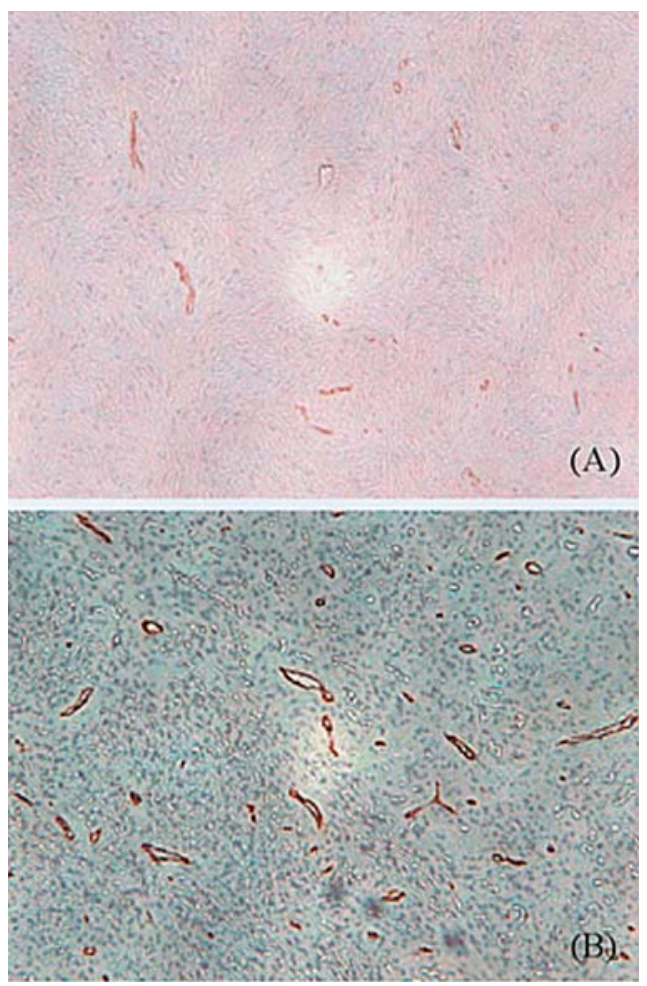

Figure 3. Immunohistochemical staining of CD31. Microvessels are visible as brown capillaries or small clusters that are distinct from the surrounding tissue. (A) Microvessel density (MVD) is low in the gastric GIST. (B) MVD is high in the small intestinal GIST.

mutations in exon 9 of the KIT gene occur preferentially in the small bowel and behave aggressively $(13,16)$. In contrast, other studies found no association of exon 9 with tumor location $(20,21)$. In the present study, the 13 extragastric GISTs, including 9 GISTs localized in the small bowel, had no mutations in exon 9 of the KIT gene.

We found mutations in exon 13 of the KIT gene in 2 extragastric GISTs. Mutations in exon 13 are thought to be rare in GISTs. To our knowledge, there are $<10$ reported sporadic GIST cases with mutations in exon 13, and all have the same K642E substitution in the KIT protein $(15,16,19)$. Chen et al (22) reported a new coincident mutation in exon 13, V654A, in STI571-resistant GISTs after treatment with STI571. Recently, we reported two substitutions in exon 13 of the KIT gene, V643A and L647P, in gastric GISTs (12). In the present study, we detected two additional substitutions, K642E and M651V, in exon 13 of the KIT gene (Fig. 2). M651V has not been detected in GISTs previously.

Corless et al (23) reported that the majority of GISTs that were $<1 \mathrm{~cm}$ in diameter contained mutations in exon 11 of the KIT gene, suggesting that KIT mutations are not size-dependent and most likely occur at an early stage in GIST development. In 4 of the 13 extragastric GISTs in the present study, we did not detect any mutations in exons $9,11,13$, and 17 of the KIT gene or exons 12 and 18 of the PDGFRA gene. We considered these four cases as wild-type. As shown in Table I, the sizes and Ki-67 LIs of these wild-type tumors were smaller than those of tumors with mutations. The mean size of the wild-type GISTs was $3.3 \mathrm{~cm}(\mathrm{p}=0.0088)$, and the mean Ki-67 LI was $1.0(\mathrm{p}=0.1277)$. There was a significant difference in tumor 
size, suggesting that wild-type GISTs are associated with non-aggressive clinical behavior.

Angiogenesis is essential for the growth, invasion, and metastasis of tumors (24). MVD is a measure of angiogenesis and is considered a strong indicator of prognosis in patients with a variety of cancers (25-30). It has been reported that MVD and expression of vascular endothelial growth factors (VEGFs), a family of angiogenic factors, are associated with poor prognosis for patients with gastric GIST $(31,32)$. Recently, Fukuta et al (33) estimated the angiogenic activity of GISTs with contrast-enhanced ultrasound (US) imaging and found that the US image is more closely correlated than histologic findings with final diagnosis. In the present study, we found that MVD in extragastric GISTs is significantly higher than that in gastric GISTs, suggesting that extragastric GIST is more aggressive than gastric GIST. However, further studies with large sample numbers and longer follow-up periods are needed.

In summary, mutations in exon 11 of the KIT gene are the most common mutations in both gastric and extragastric GISTs. However, the sizes of deleted regions (number of deleted amino acids) resulting from exon 11 mutations were larger in extragastric GISTs than in gastric GISTs. Extragastric GISTs exhibit greater angiogenesis than gastric GISTs. These results suggest that the malignant potential of GISTs in small bowel and rectum may differ from that of GISTs in the stomach.

\section{References}

1. Miettinen M, Sarlomo-Rikala M and Lasota J: Gastrointestinal stromal tumors: recent advances in understanding of their biology. Hum Pathol 30: 1213-1220, 1999.

2. Lasota J, Jasinski M, Sarlomo-Rikala M and Miettinen M: Mutations in exon 11 of $c$-Kit occur preferentially in malignant versus benign gastrointestinal stromal tumors and do not occur in leiomyomas or leiomyosarcomas. Am J Pathol 154: 53-60, 1999.

3. Miettinen $\mathrm{M}$ and Lasota J: Gastrointestinal stromal tumors definition, clinical, histological, immunohistochemical, and molecular genetic features and differential diagnosis. Virchows Arch 438: 1-12, 2001.

4. Dematteo RP, Heinrich MC, El-Rifai WM and Demetri G: Clinical management of gastrointestinal stromal tumors: before and after STI-571. Hum Pathol 33: 466-477, 2002.

5. Hirota S, Isozaki K, Moriyama Y, et al: Gain-of-function mutations of $c$-kit in human gastrointestinal stromal tumors. Science 279: 577-580, 1998

6. Vosseller K, Stella G, Yee NS and Besmer P: c-kit receptor signaling through its phosphatidylinositide-3'-kinase-binding site and protein kinase $\mathrm{C}$ : role in mast cell enhancement of degranulation, adhesion, and membrane ruffling. Mol Biol Cell 8: 909-922, 1997.

7. Sommer G, Agosti V, Ehlers I, et al: Gastrointestinal stromal tumors in a mouse model by targeted mutation of the Kit receptor tyrosine kinase. Proc Natl Acad Sci USA 100: 6706-6711, 2003.

8. Heinrich MC, Corless CL, Duensing A, et al: PDGFRA activating mutations in gastrointestinal stromal tumors. Science 299: 708-710, 2003.

9. Rossi G, Valli R, Bertolini F, et al: PDGFRA expression in differential diagnosis between KIT-negative gastrointestinal stromal tumours and other primary soft-tissue tumours of the gastrointestinal tract. Histopathology 46: 522-531, 2005.

10. Andre C, Martin E, Cornu F, Hu WX, Wang XP and Galibert F: Genomic organization of the human and c-kit gene: evolution of the receptor tyrosine kinase subclass III. Oncogene 7: 685-691, 1992.

11. Sihto H, Sarlomo-Rikala M, Tynninen O, et al: KIT and plateletderived growth factor receptor alpha tyrosine kinase gene mutations and KIT amplifications in human solid tumors. J Clin Oncol 23: 49-57, 2005.
12. Cho S, Kitadai Y, Yoshida S, et al: Deletion of the KIT gene is associated with liver metastasis and poor prognosis in patients with gastrointestinal stromal tumor in the stomach. Int J Oncol 28: 1361-1367, 2006.

13. Antonescu CR, Sommer G, Sarran L, et al: Association of KIT exon 9 mutations with nongastric primary site and aggressive behavior: KIT mutation analysis and clinical correlates of 120 gastrointestinal stromal tumors. Clin Cancer Res 9: 3329-3337, 2003.

14. Taniguchi M, Nishida T, Hirota S, et al: Effect of $c$-kit mutation on prognosis of gastrointestinal stromal tumors. Cancer Res 59: 4297-4300, 1999.

15. Kinoshita K, Isozaki K, Hirota S, et al: c-kit gene mutation at exon 17 or 13 is very rare in sporadic gastrointestinal stromal tumors. J Gastroenterol Hepatol 18: 147-151, 2003.

16. Lasota J, Wozniak A, Sarlomo-Rikala M, et al: Mutations in exon 9 and exon 13 of KIT gene are rare events in gastrointestinal stromal tumors. A study of 200 cases. Am J Pathol 157: 1091-1095, 2000.

17. Ernst SI, Hubbs AE, Przygodzki RM, Emory TS, Sobin LH and O'Leary TJ: KIT mutation portends poor prognosis in gastrointestinal stromal/smooth muscle tumors. Lab Invest 78 : 1633-1636, 1998.

18. Moskaluk CA, Tian Q, Marshall CR, Franquemont DW, Rumpel CA and Frierson HF Jr: Mutations of $c$-kit JM domain are found in a minority of human gastrointestinal stromal tumors. Oncogene 18: 1897-1902, 1999.

19. Lux ML, Rubin BP, Biase TL, et al: KIT extracellular and kinase domain mutations in gastrointestinal stromal tumors. Am J Pathol 156: 791-795, 2000.

20. Hirota S, Nishida T, Isozaki K, et al: Gain-of-function mutation at the extracellular domain of KIT in gastrointestinal stromal tumours. J Pathol 193: 505-510, 2001.

21. Sakurai S, Oguni S, Hironaka M, Fukayama M, Morinaga S and Saito K: Mutations in c-kit gene exons 9 and 13 in gastrointestinal stromal tumors among Japanese. Jpn J Cancer Res 92: 494-498, 2001

22. Chen LL, Trent JC, Wu EF, et al: A missense mutation in KIT kinase domain 1 correlates with imatinib resistance in gastrointestinal stromal tumors. Cancer Res 64: 5913-5919, 2004.

23. Corless CL, McGreevey L, Haley A, Town A and Heinrich MC: KIT mutations are common in incidental gastrointestinal stromal tumors one centimeter or less in size. Am J Pathol 160: 1567-1572, 2002.

24. Folkman J: How is blood vessel growth regulated in normal and neoplastic tissue? G.H.A. Clowes Memorial Award lecture. Cancer Res 46: 467-473, 1986.

25. Macchiarini P, Fontanini G, Hardin MJ, Squartini F and Angeletti CA: Relation of neovascularisation to metastasis of non-small-cell lung cancer. Lancet 340: 145-146, 1992.

26. Weidner N, Carroll PR, Flax J, Blumenfeld W and Folkman J: Tumor angiogenesis correlates with metastasis in invasive prostate carcinoma. Am J Pathol 143: 401-409, 1993.

27. Toi M, Kashitani J and Tominaga T: Tumor angiogenesis is an independent prognostic indicator in primary breast carcinoma. Int J Cancer 57: 371-374, 1993.

28. Porschen R, Classen S, Piontek M and Borchard F: Vascularization of carcinomas of the esophagus and its correlation with tumor proliferation. Cancer Res 54: 587-591, 1994.

29. Hollingsworth HC, Kohn EC, Steinberg SM, Rothenberg ML and Merino MJ: Tumor angiogenesis in advanced stage ovarian carcinoma. Am J Pathol 147: 33-41, 1995.

30. Maeda K, Chung YS, Takatsuka S, et al: Tumour angiogenesis and tumour cell proliferation as prognostic indicators in gastric carcinoma. Br J Cancer 72: 319-323, 1995.

31. Takahashi R, Tanaka S, Kitadai Y, et al: Expression of vascular endothelial growth factor and angiogenesis in gastrointestinal stromal tumor of the stomach. Oncology 64: 266-274, 2003.

32. Chen WT, Huang CJ, Wu MT, Yang SF, Su YC and Chai CY: Hypoxia-inducible factor- $1 \alpha$ associated with risk of aggressive behavior and tumor angiogenesis in gastrointestinal stromal tumor. Jpn J Clin Oncol 35: 207-213, 2005.

33. Fukuta N, Kitano M, Maekawa K, Chikugo T and Kudo M: Estimation of the malignant potential of gastrointestinal stromal tumors: the value of contrast-enhanced coded phase-inversion harmonic US. J Gastroenterol 40: 247-255, 2005. 